\title{
MicroRNA-137-regulated AKT serine/threonine kinase 2 inhibits tumor growth and sensitizes cisplatin in patients with non-small cell lung cancer
}

\author{
ZHUMING LU ${ }^{1}$, MINGHUI WANG ${ }^{2}$, SHUOYUN WU ${ }^{1}$, MIN YE ${ }^{1}$, \\ ZHICHAO LIN ${ }^{1}$, TAO SHUN ${ }^{1}$ and CHUXIAO DUAN ${ }^{1}$ \\ ${ }^{1}$ Department of Thoracic Surgery, Jiangmen Central Hospital, Sun Yat-Sen University, \\ Jiangmen, Guangdong 529030; ${ }^{2}$ Department of Thoracic Surgery, Sun Yat-Sen Memorial Hospital, \\ Sun Yat-Sen University, Guangzhou, Guangdong 520120, P.R. China
}

Received August 3, 2016; Accepted January 22, 2018

DOI: $10.3892 / \mathrm{ol} .2018 .8823$

\begin{abstract}
The present study investigated the role of microRNA-137-regulated AKT serine/threonine kinase 2 (AKT2) on tumor growth and cisplatin sensitivity in patients with non-small cell lung cancer (NSCLC). The results demonstrated that the expression of microRNA-137 in cisplatin-treated NSCLC patient tissue samples was markedly lower than that in healthy tissue samples. The disease-free survival and overall survival rates of patients with NSCLC exhibiting a high microRNA-137 expression were higher than the survival rates of patients with NSCLC exhibiting a low expression of microRNA-137. Overexpression of microRNA-137 inhibited the proliferation of A549 and H520 cells treated with cisplatin. Overexpression of miR-137 suppressed the protein expression of AKT2, increased caspase-3 activity, increased Bax protein expression and suppressed Cyclin D1 protein expression in A549 and H520 cells treated with cisplatin. MK2206, an AKT2 inhibitor, inhibited AKT2 protein expression and suppressed the proliferation of A549 and H520 cells treated with cisplatin following overexpression of miR-137. The inhibition of AKT2 also increased caspase-3 activity and Bax protein expression, and suppressed Cyclin D1 protein expression in A549 and H520 cells treated with cisplatin following overexpression of miR-137. Taken together, the results of the present study suggested that microRNA-137-regulated AKT2 inhibits tumor growth and sensitizes cisplatin in patients with NSCLC.
\end{abstract}

Correspondence to: Dr Zhuming Lu, Department of Thoracic Surgery, Jiangmen Central Hospital, Sun Yat-Sen University, 23 Haibang Street, Jiangmen, Guangdong 529030, P.R. China E-mail: zhibengraochuang74@126.com

Key words: microRNA-137, AKT serine-/threonine kinase 2, cisplatin, non-small cell lung cancer

\section{Introduction}

Lung cancer is a malignant tumor with the highest rate of cancer-associated mortality globally (1). Non-small cell lung cancer (NSCLC) is the most common form of lung cancer and accounts for $>70 \%$ of all lung cancer cases (1). According to a recent survey undertaken by the World Health Organization, the incidence of lung cancer is $33.5 / 100,000$, including (45.9/10,000 males and 21.3/100,000 females), with the incidence in males being markedly higher than that in females (2). With the increasing progress of surgery and other therapeutic alternatives, treatment options have already greatly improved the prognosis and quality of life of patients with lung cancer (3). However, due to the fact that there is a lack of in-depth knowledge regarding the molecular mechanisms of lung cancer morbidity, early diagnosis targets and anticancer treatments for patients with lung cancer are limited (3). It has reported that, by the time patients with NSCLC see a doctor, $>50 \%$ of cases have already developed into (at least) stage III disease, meaning that surgical treatment is no longer an option (4). In recent years, chemotherapy is regarded as one of the most effective method for curing more advanced NSCLC (5). However, cancer is a highly heterogeneous disease. In patients with the same pathological type of lung at the point of diagnosis, there is a marked difference in the sensitivity of platinum chemotherapeutics (1).

At present, it is widely believed that different genotypes in patients with NSCLC are associated with different degrees of sensitivity to platinum-based chemotherapeutics (6). Platinum-based chemotherapeutics, including cis-platinum and carboplatin, are widely applied as anticancer chemotherapeutics. Furthermore, the curative effects of these agents are the most efficient and they are more cytotoxic (7). Once platinum drugs enter into the nucleus and combine with DNA, they cause irreversible damage to DNA and induce apoptosis by forming platinum-DNA complexes, in order to induce their anti-carcinogenic action (8). Due to the fact that there are DNA damage repair mechanisms in cells, DNA damage caused by platinum-based chemotherapeutics may be repaired by increasing the abundance of factors associated with DNA 
damage repair (9). Therefore, it is possible that the expression and function of regulatory factors in the DNA repair process serves an important role in the sensitivity of tumors to platinum-based chemotherapeutics (10). The expression of these molecules and the abnormal activation of their functions may impact the effects of platinum-based chemotherapeutics on patients with NSCLC by enhancing the tolerance of cancer cells to platinum-based chemotherapeutics (11).

MicroRNAs (miRNAs/) are short-chain, non-coding RNAs, which develop their biological functions through multiple mechanisms in order to control protein expression. In a previous study, the results of bioinformatics analysis have demonstrated that every miRNA is able to control several hundreds of gene targets and to participate in a conduction group of multiple gene signaling paths (12). Therefore, miRNAs control a series of biological functions, including cell proliferation, differentiation and apoptosis (13). Altered expression of miRNAs may have a huge influence on the functional activity of cells. Genomic research of human cancer has revealed that miRNA expression is varied (14). A lot of diagnosis and prognosis-based biological information are associated with miRNA expression (14). Therefore, miRNA expression may predict the prognosis of NSCLC, as an imbalance in miRNA expression frequently occurs in all types of tumor. Therefore, these expression characteristics have potential value in the diagnosis and prognosis of tumors (15).

AKT, also known as protein kinase B (PKB), is a serine/ threonine protein kinase and therefore, it is highly homologous with protein kinase A (PKA) and PKC (16). AKT serves an important role in tumor cell proliferation, differentiation, invasion and metabolism (17). AKT2 is an important subtype of AKT and, as an oncogene, may cause malignant changes to cells (18). It has been revealed that AKT2 is overexpressed or has increased activity in ovarian cancer, breast cancer, glioma and other types of tumor (18). Furthermore, AKT2 is associated with tumor proliferation, invasion, metastasis and prognosis. At present, studies regarding AKT2 expression in NSCLC are insufficient. The present study demonstrated that microRNA-137 inhibits tumor growth and sensitizes tumor cells to cisplatin in patients with non-small cell lung cancer through AKT2.

\section{Materials and methods}

Clinical specimens and cell culture. NSCLC and adjacent non-cancerous tissues from 79 cisplatin-treated patients (age range, $65.5 \pm 8.5$ years old, male) were obtained during surgical resection in Jiangmen Central Hospital (Jiangmen, China) between March 2015 and May 2015. The experimental protocols were approved by the Ethics Committees of Jiangmen Central Hospital and written informed consent was obtained from all participants. The patients were followed up every month to record the disease-free survival (DFS) and overall survival (OS) rates. Human lung cancer A549 and H520 cell lines were maintained in RPMI-1640 medium (Gibco; Thermo Fisher Scientific, Inc., Waltham, MA, USA), supplemented with $10 \%$ fetal bovine serum (Hyclone; Logan, UT, USA), $100 \mathrm{U} / \mathrm{ml}$ penicillin and $100 \mathrm{mg} / \mathrm{ml}$ streptomycin in a humidified atmosphere of $5 \% \mathrm{CO}_{2}$ and $95 \% \mathrm{O}_{2}$ at $37^{\circ} \mathrm{C}$.
RNA extraction and reverse transcription-quantitative polymerase chain reaction $(R T-q P C R)$. Total RNA was extracted from tissues using TRIzol reagent (Invitrogen; Thermo Fisher Scientific, Inc.), according to the manufacturer's protocol. Total RNA was reversely transcribed with oligodT primers using PrimeScript RT Reagent kit (Vazyme, Piscataway, NJ, USA) at $37^{\circ} \mathrm{C}$ for $60 \mathrm{~min}$ and at $85^{\circ} \mathrm{C}$ for $1 \mathrm{~min}$. The cDNA was amplified by RT-qPCR using SYBR ${ }^{\circledR}$ Premix Ex Taq (Takara Biotechnology Co., Ltd., Dalian, China). PCR thermocycling conditions were as follows: $5 \mathrm{~min}$ at $95^{\circ} \mathrm{C}, 40$ cycles of $30 \mathrm{sec}$ at $95^{\circ} \mathrm{C}, 60 \mathrm{sec}$ at $60^{\circ} \mathrm{C}, 30 \mathrm{sec}$ at $72^{\circ} \mathrm{C}$. Experiments were performed in triplicate. The primer sequences were as follows: microRNA-137 forward, 3'-GCT CCTCAGGTCGAACCTATTG-5' and reverse, 3'-CCGACG CTATTGCTTAAGAATACG-5'; U6 Forward: 5'-CGCTTC GGCAGCACATATACTAAAATTGGAAC-3' and reverse: 5'-GCTTCACGAATTTGCGTGTCATCCTTGC-3'. The expression of microRNA-137 was calculated by relative quantification using the $2^{-\Delta \Delta C q}$ method (19). Expression of microRNA-137 was considered to be low at $<50 \%$ of U6 expression, and high at 50-100\% of U6 expression,

Transfection and treatment with the AKT2 inhibitor, MK2206. Negative control (pLV3-control) and microRNA-137 mimic (pLV3-microRNA-137 mimic) were constructed by Sangon Biotech Co., Ltd., (Shanghai, China). Negative control (100 ng) and microRNA-137 mimic (100 ng) were transfected into A549 and H520 cells using Lipofectamine 2000 (Invitrogen; Thermo Fisher Scientific, Inc.) at $37^{\circ} \mathrm{C}$. Following transfection for $4 \mathrm{~h}$, the medium was refreshed and $1.25 \mu \mathrm{M}$ cisplatin was added to the cells at $37^{\circ} \mathrm{C}$. Cells were treated with cisplatin at 12, 24 and $48 \mathrm{~h}$ for MTT, and treated with cisplatin at $48 \mathrm{~h}$ for Caspase-3 activity and western blot analysis. Next, $2.5 \mu \mathrm{M}$ MK2206 and $1.25 \mu \mathrm{M}$ cisplatin were added to the cells following transfection for $4 \mathrm{~h}$ at $37^{\circ} \mathrm{C}$.

Cell proliferation. A549 and H520 cells transfected with microRNA-137 or anti-microRNA-137 plasmids were seeded onto 96-well plates at a density of 4,000 cells/well and were treated with $1.25 \mu \mathrm{M}$ cisplatin for 12,24 or $48 \mathrm{~h}$. A total of $10 \mu 1$ MTT (Invitrogen; Thermo Fisher Scientific, Inc.) was added to each well, followed by incubated for $4 \mathrm{~h}$ in a humidified atmosphere of $5 \% \mathrm{CO}_{2}$ and $95 \% \mathrm{O}_{2}$ at $37^{\circ} \mathrm{C}$. A total of $200 \mu$ l dimethyl sulfoxide was added to each well to dissolve the purple formazan, after the supernatant had been discarded. The optical density (OD) was detected at a wavelength of $490 \mathrm{~nm}$ using a POLARstar OPTIMA multi-detection microplate reader (Bio-Rad Laboratories, Inc., Hercules, CA, USA).

Caspase-3 activity. A549 and H520 cells transfected with microRNA-137 or anti-microRNA-137 plasmids were seeded onto 6-well plates at a density of $1 \times 10^{6}$ cells/well and were treated with $1.25 \mu \mathrm{M}$ cisplatin for $24 \mathrm{~h}$. Ac-DEVD-pNA was added to each well, followed by incubation for $1 \mathrm{~h}$ in a humidified atmosphere of $5 \% \mathrm{CO}_{2}$ and $95 \% \mathrm{O}_{2}$ at $37^{\circ} \mathrm{C}$. The OD was detected at a wavelength of $405 \mathrm{~nm}$ using a POLARstar OPTIMA multi-detection microplate reader (Bio-Rad Laboratories, Inc.).

Western blot analysis. A549 and H520 cells transfected with microRNA-137 or anti-microRNA-137 plasmids were seeded 
A

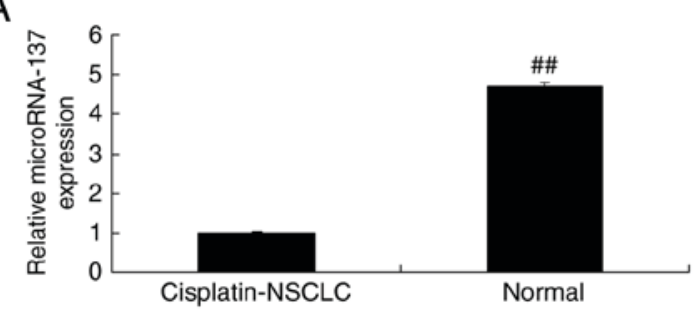

B

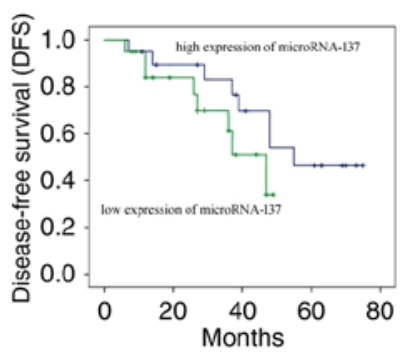

$\mathrm{C}$

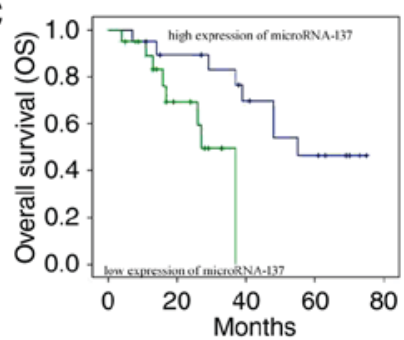

Figure 1. Expression of microRNA-137, and the DFS and OS rates of patients with NSCLC. (A) microRNA-137 expression. (B) DFS and (C) OS rates of patients with NSCLC. ${ }^{\#} \mathrm{P}<0.01$, compared with the cisplatin-treated NSCLC patient tissue group. Cisplatin-NSCLC, cisplatin-treated NSCLC patient tissue; Normal, adjacent healthy tissue. NSCLC, non-small cell lung cancer; DFS, disease-free survival; OS, overall survival.

onto 6-well plates at a density of $1 \times 10^{6}$ cells/well and were treated with $1.25 \mu \mathrm{M}$ cisplatin for $24 \mathrm{~h}$. Cells were washed twice with phosphate-buffered saline and were prepared using radioimmunoprecipitation assay buffer (Beyotime Institute of Biotechnology, Haimen, China), supplemented with phenylmethylsulfonyl fluoride (Beyotime Institute of Biotechnology). The supernatants were collected and the protein concentration was determined using a bicinchoninic acid assay (Beyotime Institute of Biotechnology). Protein $(60 \mu \mathrm{g})$ was separated by $10 \%$ SDS-PAGE and transferred onto polyvinylidene difluoride membranes (Millipore). The membranes were blocked using 5\% skimmed milk powder in TBST for $1 \mathrm{~h}$ at $37^{\circ} \mathrm{C}$, and were incubated with primary antibodies against Bax (cat no. sc-6236; 1:1,000; Santa Cruz Biotechnology, Inc., Dallas, TX, USA), Cyclin D1 (cat no. sc-717; 1:1,000; Santa Cruz Biotechnology, Inc.), p-AKT2 (cat no. sc-7985-R; 1:1,000; Santa Cruz Biotechnology, Inc.) and GAPDH (cat no. sc-25778; 1:2,000; Santa Cruz Biotechnology, Inc.) at $4^{\circ} \mathrm{C}$ overnight. The membranes were washed with TBST and incubated in a solution containing a goat anti-rabbit IgG horseradish peroxidase-conjugated secondary antibody (cat no. sc-2004; 1:5,000; Santa Cruz Biotechnology, Inc.) for $1 \mathrm{~h}$ at $37^{\circ} \mathrm{C}$. An enhanced chemiluminescence detection system (Thermo Fisher Scientific, Inc.) was used for signal detection and analyzed using ImageJ 3.0 software (Bio-Rad Laboratories, Inc.).

Statistical analysis. All data are expressed as the mean \pm standard deviation. A two-tailed Student's t-test or one-way analysis of variance by Tukey's post-hoc test were used for statistical analyses. $\mathrm{P}<0.05$ was considered to indicate a statistically significant difference.

\section{Results}

Association between microRNA-137 expression and the DFS and OS of patients with NSCLC. The microRNA-137 expression in cisplatin-treated NSCLC patient tissue samples and adjacent healthy tissue samples was measured, and it was revealed that the expression of microRNA-137 in cisplatin-treated NSCLC patient tissue samples was markedly lower than that in the adjacent healthy tissue samples (Fig. 1A). Furthermore, the DFS and OS rates in patients with NSCLC exhibiting a high expression of microRNA-137 were lower compared with those in patients with NSCLC exhibiting a low expression of microRNA-137 (Fig. 1B and C).
Effect of microRNA-137 overexpression on cell proliferation and caspase-3 activity in cisplatin-treated A549 and H52O cells. In order to determine the effect of microRNA-137 on cell proliferation and caspase-3 activity in A549 and H520 cells treated with cisplatin or transfected with microRNA-137 mimics (to induce microRNA-137 overexpression). The results demonstrated that overexpression of microRNA-137 significantly inhibited the proliferation of A549 and H520 cells treated with cisplatin compared with A549 cells expressing negative control (Fig. 2A and B). Overexpression of microRNA-137 significantly increased caspase-3 activity in A549 and H520 cells treated with cisplatin compared with those transfected with microRNA-Negative (Fig. 2C and D).

Effect of microRNA-137 overexpression on Bax protein expression in A549 and H520 cells treated with cisplatin. In order to investigate the effect of microRNA-137 on the apoptosis of A549 and H520 cells treated with cisplatin, Bax protein expression was measured using western blot analysis following overexpression of microRNA-137. The results demonstrated that overexpression of microRNA-137 significantly increased the expression of Bax protein in A549 and H520 cells treated with cisplatin compared with those transfected with microRNA-Negative (Fig. 3).

Effect of microRNA-137 overexpression on Cyclin D1 protein expression in A549 and H520 cells treated with cisplatin. In order to determine the association between microRNA-137 expression levels and Cyclin D1 expression in cisplatin-treated patients with NSCLC, western blot analysis was used to measure the protein expression of Cyclin D1 in A549 and H520 cells treated with cisplatin. Compared with the cells transfected with microRNA-Negative, Cyclin D1 protein expression was significantly inhibited in cisplatintreated A549 and H520 cells following overexpression of microRNA-137 (Fig. 4).

Effect of microRNA-137 overexpression on AKT2 protein expression in A549 and H520 cells treated with cisplatin. In order to identify any association between microRNA-137 expression and p-AKT2 expression in cisplatin-treated patients with NSCLC, p-AKT2 protein expression was determined using western blot analysis. The results demonstrated that p-AKT2 protein expression was significantly suppressed by overexpression of microRNA-137 compared with transfection with microRNA-Negative (Fig. 5). 

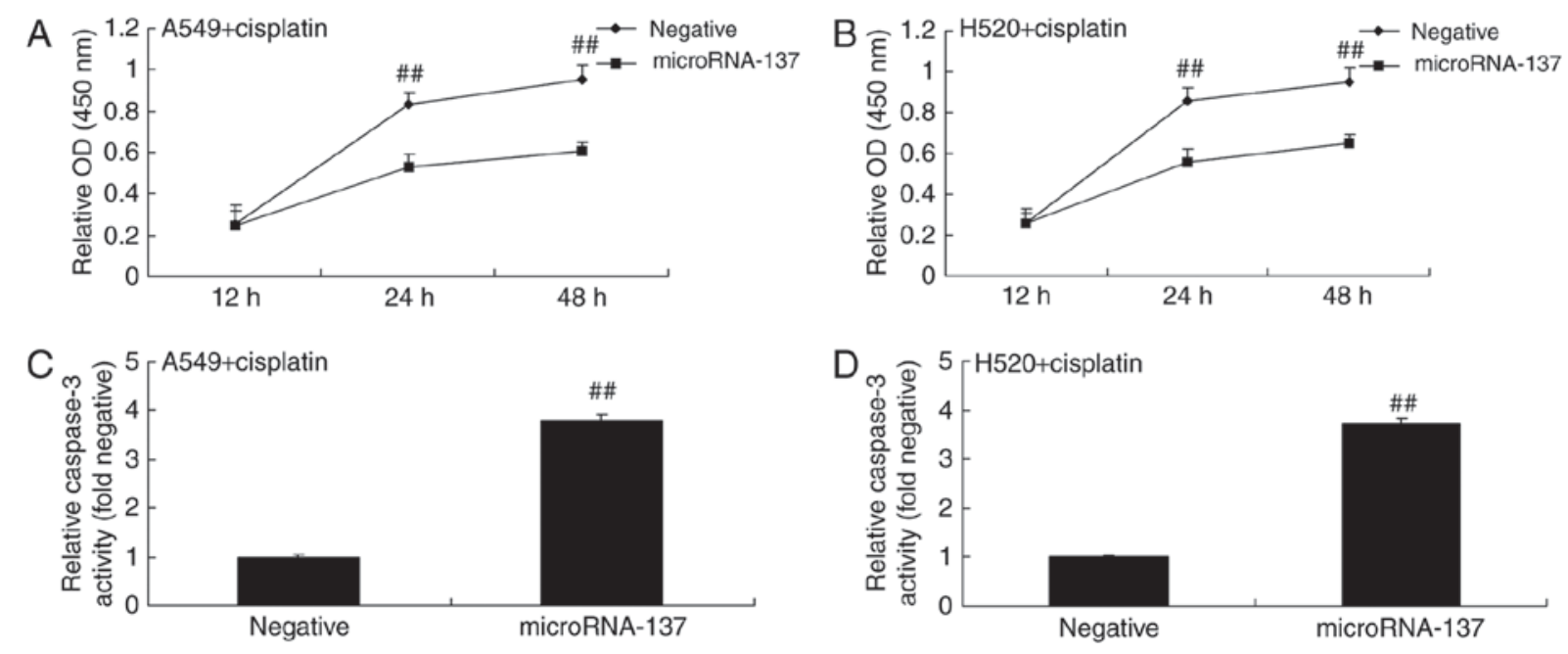

Figure 2. Effect of microRNA-137 overexpression on the cell proliferation and caspase-3 activity of A549 and H520 cells treated with cisplatin. The effect of microRNA-137 overexpression on the proliferation of (A) A549 and (B) H250 cells treated with cisplatin. The effect of microRNA-137 overexpression on the caspase-3 activity of (C) A549 and (D) H520 cells treated with cisplatin. ${ }^{\# \#} \mathrm{P}<0.01$ compared with the microRNA-Negative group. Negative, negative control group; microRNA-137, microRNA-137 group.
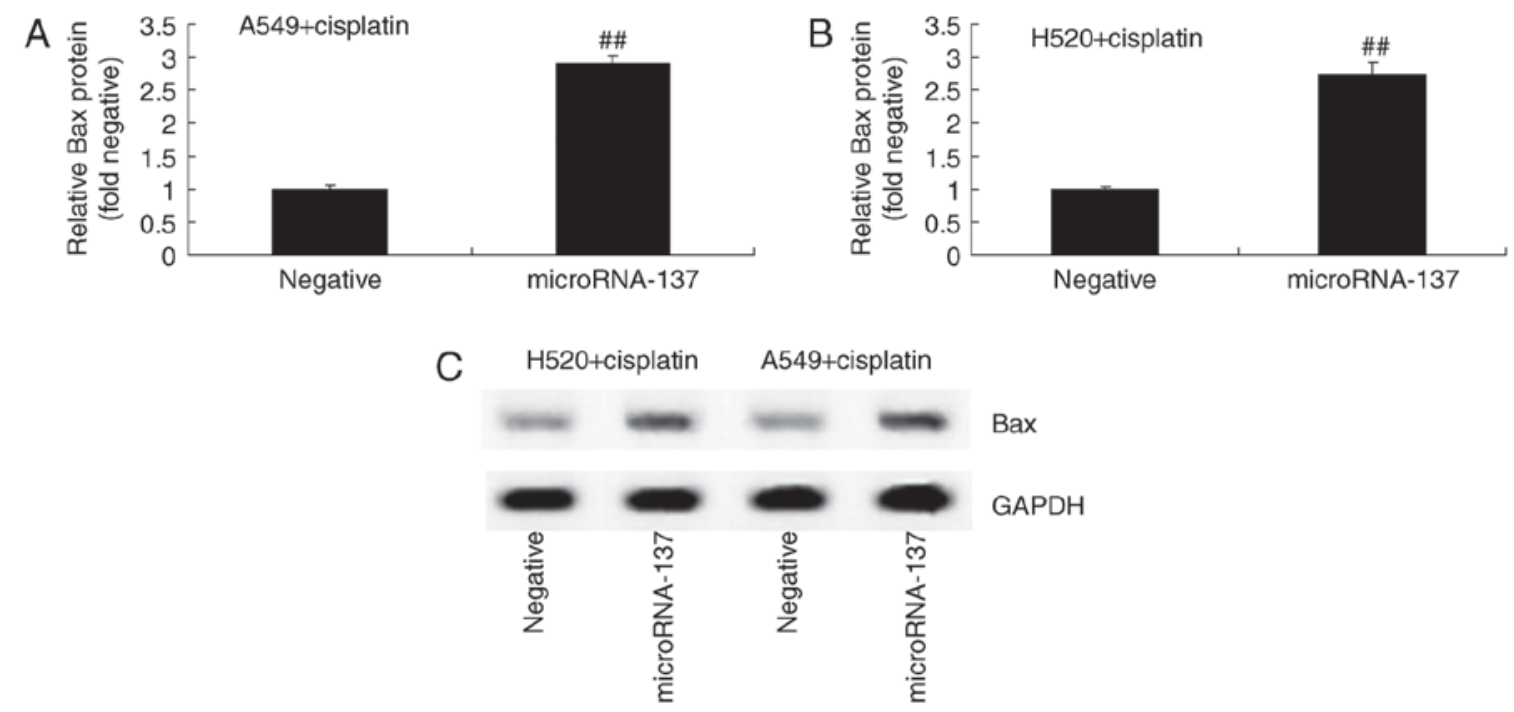

Figure 3. Effect of microRNA-137 overexpression on Bax protein expression in A549 and H520 cells treated with cisplatin. Densitometric analysis of the effect of microRNA-137 overexpression on the Bax protein expression of (A) A549 and (B) H520 cells treated with cisplatin by statistical analysis. (C) Western blot analysis of Bax protein expression. ${ }^{\# \#} \mathrm{P}<0.01$ compared with the microRNA-Negative group. Negative, negative control group; microRNA-137, microRNA-137 group.

Effect of an AKT2 inhibitor on AKT2 protein expression in $A 549$ and H520 cells treated with cisplatin following downregulation of microRNA-137. Furthermore, the effect of suppression of AKT2 (MK2206) on AKT2 protein expression in A549 and H520 cells treated with cisplatin following downregulation of microRNA-137. As demonstrated in Fig. 6, MK2206, an AKT2 inhibitor, inhibited p-AKT2 protein expression in A549 and H520 cells treated by cisplatin following downregulation of microRNA-137 compared with cells transfected with microRNA-Negative.

Effect of an AKT2 inhibitor on cell proliferation and caspase-3 activity in A549 and H520 cells treated with cisplatin following downregulation of microRNA-137. The present study investigated whether or not the AKT2 inhibitor had an effect on cell proliferation and caspase-3 activity in A549 and H520 cell treated with cisplatin following downregulation of microRNA-137. The AKT2 inhibitor significantly suppressed the proliferation of A549 and H520 cells treated with cisplatin following downregulation of microRNA-137 compared with those transfected with microRNA-Negative (Fig. 7A and B). The suppression of AKT2 significantly increased caspase-3 activity in A549 and H520 cells treated with cisplatin following downregulation of microRNA-137 compared with those transfected with microRNA-Negative (Fig. 7C and D).

Effect of an AKT2 inhibitor on Bax protein expression in A549 and $H 520$ cells treated with cisplatin following downregulation of microRNA-137. The present study investigated the effect 

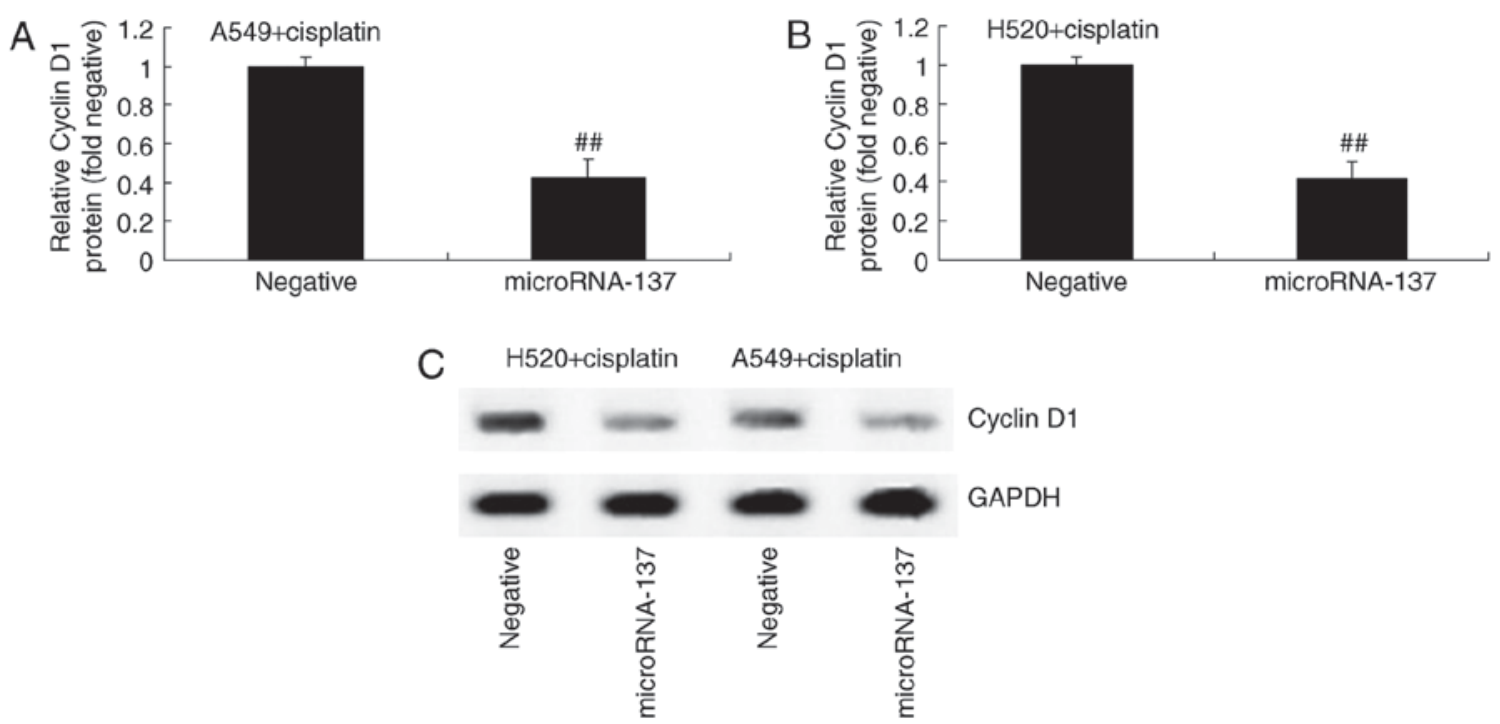

Figure 4. Effect of microRNA-137 overexpression on Cyclin D1 protein expression in A549 and H520 cells treated with cisplatin. Densitometric analysis of the effect microRNA-137 overexpression on Cyclin D1 protein expression in (A) A549 and (B) H520 cells treated with cisplatin. (C) Western blot analysis of Cyclin D1 protein expression. ${ }^{\#} \mathrm{P}<0.01$ compared with microRNA-Negative group. Negative, negative control group; microRNA-137, microRNA-137 group.
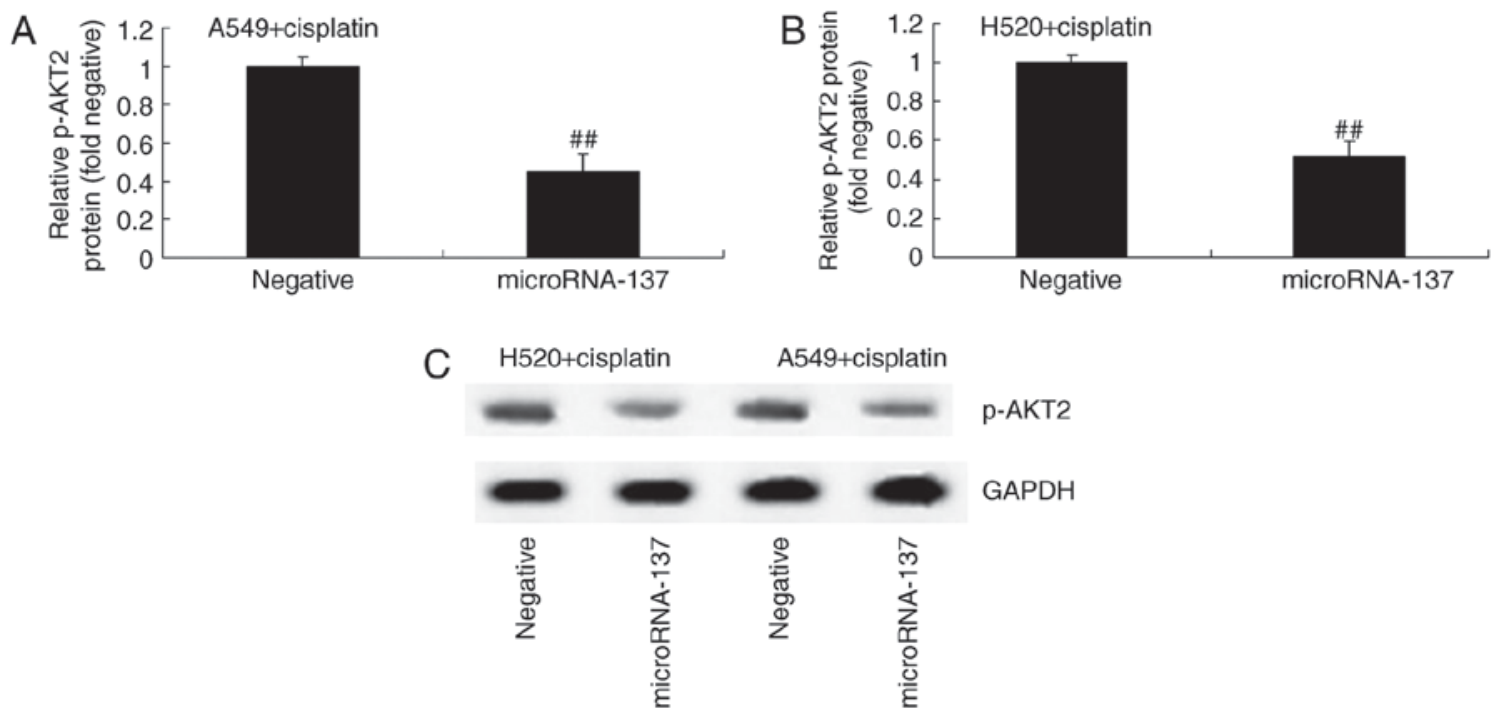

Figure 5. Effect of microRNA-137 overexpression on AKT2 protein expression in A549 and H520 cells treated with cisplatin. Densitometric analysis of the effect of microRNA-137 overexpression on p-AKT2 protein expression in (A) A549 and (B) H520 cells treated with cisplatin. (C) Western blot analysis of p-AKT2 protein expression. ${ }^{\# \#} \mathrm{P}<0.01$ compared with microRNA-Negative group. Negative, negative control group; microRNA-137, microRNA-137 group.

of AKT2 inhibition on Bax protein expression in A549 and H520 cells treated with cisplatin following downregulation of microRNA-137. The results demonstrated that the suppression of AKT2 significantly increased Bax protein expression in A549 and H520 cells treated with cisplatin following downregulation of microRNA-137, compared with cells transfected with microRNA-Negative (Fig. 8).

Effect of an AKT2 inhibitor on Cyclin D1 protein expression in A549 and H520 cells treated with cisplatin following downregulation of microRNA-137. The effect of AKT2 suppression on Cyclin D1 protein expression in A549 and H520 cells treated with cisplatin following downregulation of microRNA-137 was investigated. Downregulation of microRNA-137 significantly suppressed the protein expression of Cyclin D1 in A549 and H520 cells treated with cisplatin (Fig. 9).

\section{Discussion}

Lung cancer is regarded as a malignant tumor with the highest rate of morbidity and mortality of all types of cancer. NSCLC is the most common type of lung cancer, accounting for $>70 \%$ of all lung cancer cases (20). At present, a multidisciplinary treatment strategy is employed for the treatment of NSCLC (4). Platinum-based chemotherapeutics, including cis-platinum and carboplatin, are widely applied anticancer chemotherapeutics (4). Furthermore, these agents also exhibit the most efficient curative effects and are more cytotoxic (5). The 
A

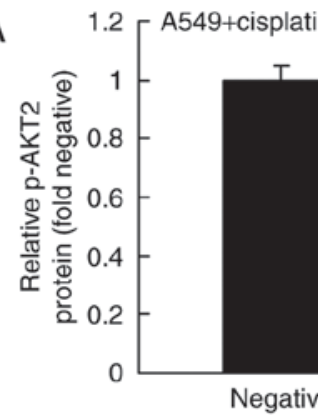

Platin

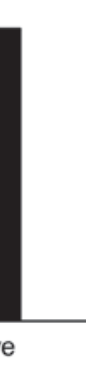

C

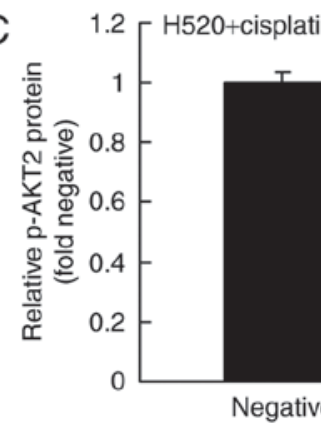

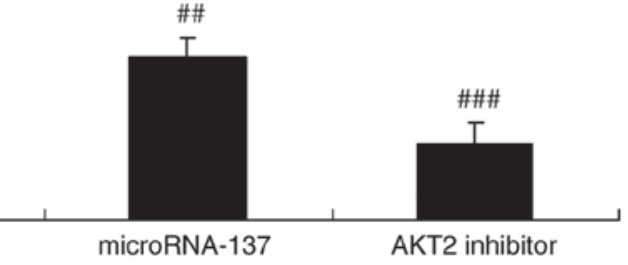

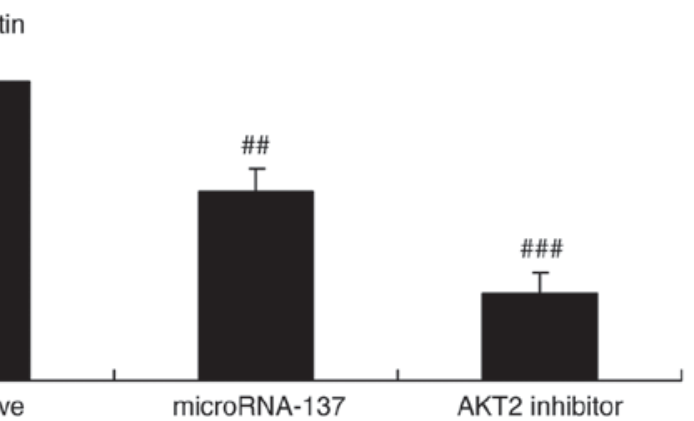

B

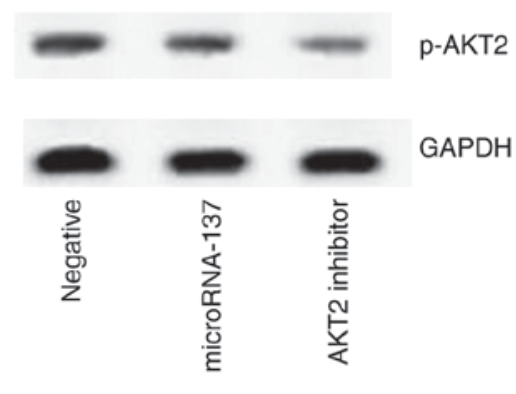

D

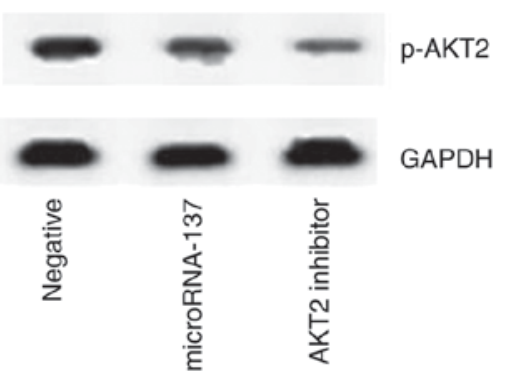

Figure 6. Effect of an AKT2 inhibitor on AKT2 protein expression in A549 and H520 cell treated with cisplatin following downregulation of microRNA-137. (A) Densitometric and (B) western blot analysis of the effect on an AKT2 inhibitor on AKT2 protein expression in A549 cells treated with cisplatin following downregulation of microRNA-137. (C) Densitometric and (D) western blot analysis of the effect on an AKT2 inhibitor on AKT2 protein expression in H520 cells treated with cisplatin following downregulation of microRNA-137. ${ }^{\# \#} \mathrm{P}<0.01$ compared with microRNA-Negative group; ${ }^{\# \#} \mathrm{P}<0.01$ compared with microRNA-137 group. Negative, negative control group; microRNA-137, microRNA-137 group; AKT2 inhibitor, microRNA-137+MK2206 group.
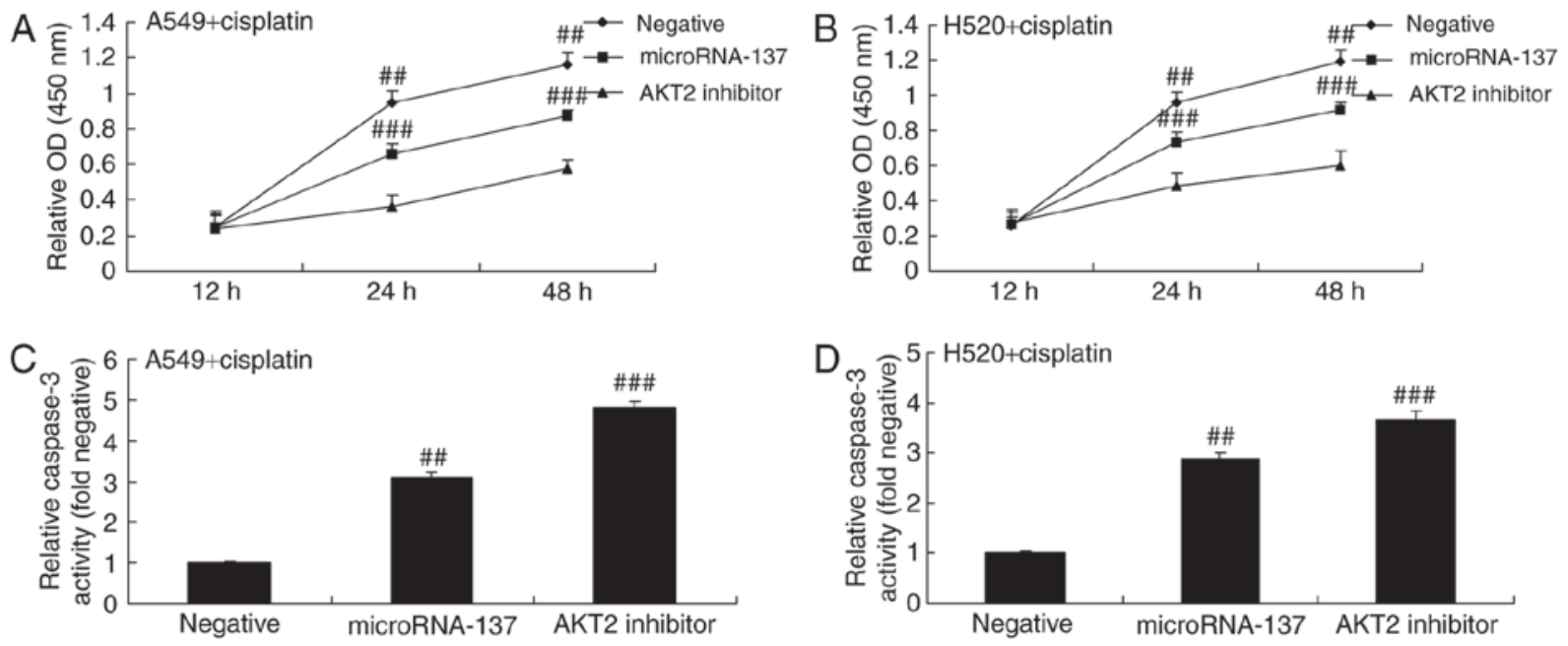

Figure 7. Effect of an AKT2 inhibitor on the cell proliferation and caspase-3 activity of A549 and H520 cells treated with cisplatin following downregulation of microRNA-137. The effect of an AKT2 inhibitor on the proliferation of (A) A549 and (B) H520 cells. The effect of an AKT2 inhibitor on the caspase-3 activity of (C) A549 and (D) H520 cells treated with cisplatin following downregulation of microRNA-137. ${ }^{\# \prime}$ P $<0.01$ compared with microRNA-Negative group; ${ }^{\# \# \# P<0.01 ~ c o m p a r e d ~ w i t h ~ m i c r o R N A-137 ~ g r o u p . ~ N e g a t i v e, ~ n e g a t i v e ~ c o n t r o l ~ g r o u p ; ~ m i c r o R N A-137, ~ m i c r o R N A-137 ~ g r o u p ; ~ A K T 2 ~ i n h i b i t o r, ~}$ microRNA-137+MK2206 group.

present study revealed that the expression of microRNA-137 in cisplatin-treated NSCLC patient tissue samples was significantly lower than that of normal tissue samples.

Expression of microRNA-137 in adult brain glioma is markedly reduced and may even participate in the morbidity of schizophrenia by regulating the maturity of neurons in the nervous system (21). microRNA-137 serves a function in numerous diseases by participating in the regulation of the cell cycle and cell differentiation (22). The results of the present study demonstrated that the DFS and OS rates of patients with NSCLC exhibiting a high expression of microRNA-137 were higher than those of patients with NSCLC exhibiting a low expression of microRNA-137. Additionally, it was also revealed that overexpression of microRNA-137 significantly inhibited cell proliferation and increased caspase-3 activity in A549 and H520 cells treated with cisplatin.

Previous studies have demonstrated that the development of lung cancer involves multiple factors, the formation of 
A

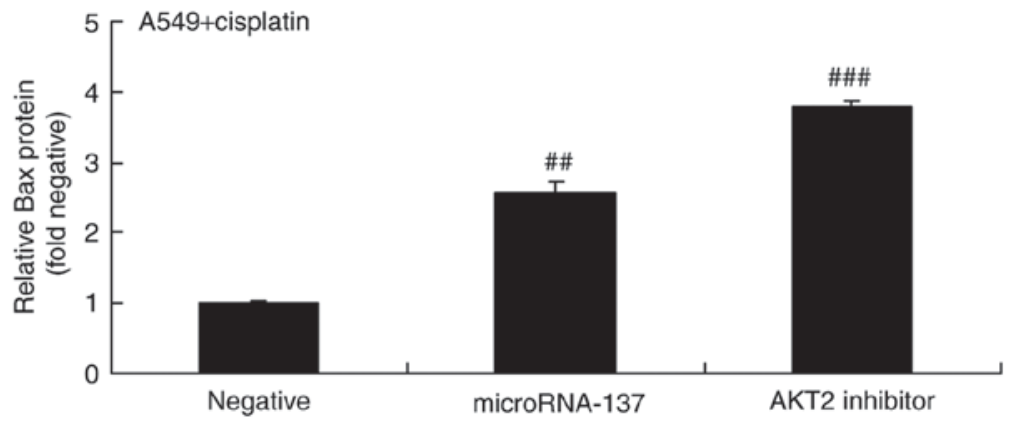

C

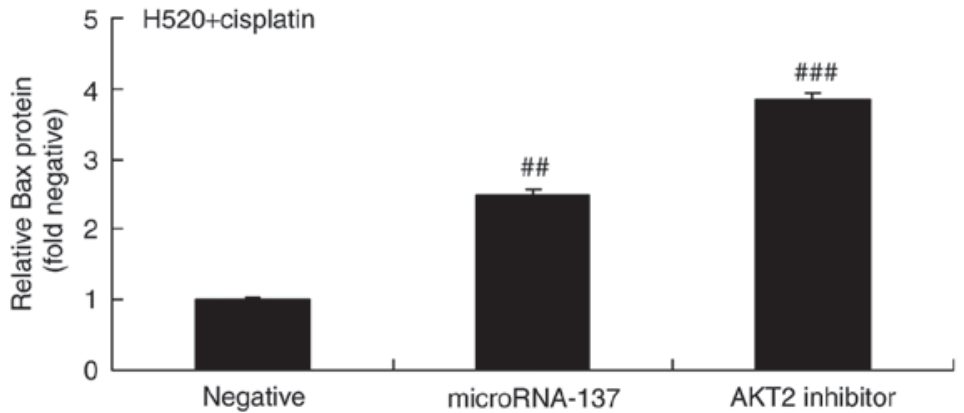

B

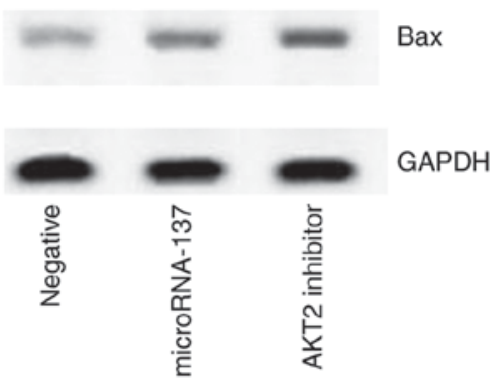

D

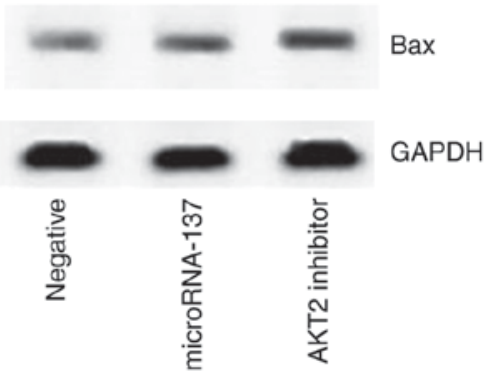

Figure 8. Effect of an AKT2 inhibitor on Bax protein expression in A549 and H520 cells treated with cisplatin following downregulation of microRNA-137. (A) Densitometric and (B) western blot analysis of the effect of an AKT2 inhibitor on Bax protein expression in A549 cells treated with cisplatin following downregulation of microRNA-137. (C) Densitometric and (D) western blot analysis of the effect of an AKT2 inhibitor on Bax protein expression in H520 cells treated with cisplatin following downregulation of microRNA-137. ${ }^{\# \#} \mathrm{P}<0.01$ compared with microRNA-Negative group; ${ }^{\# \# \#} \mathrm{P}<0.01$ compared with microRNA-137 group. Negative, negative control group; microRNA-137, microRNA-137 group; AKT2 inhibitor, microRNA-137+MK2206 group.

A

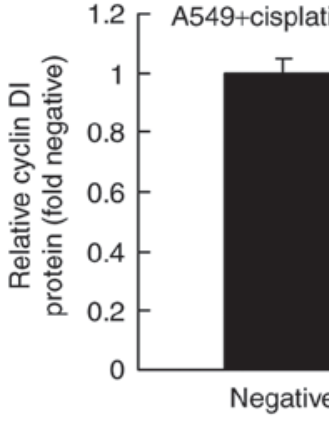

$\mathrm{B}$

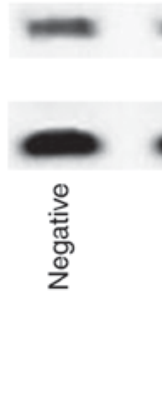

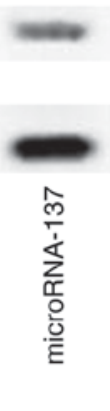

D

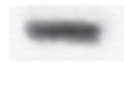

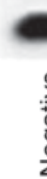

Cyclin DI

GAPDH

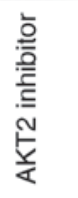

C

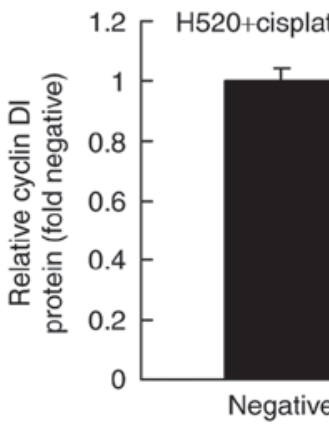

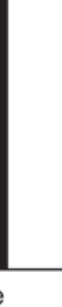

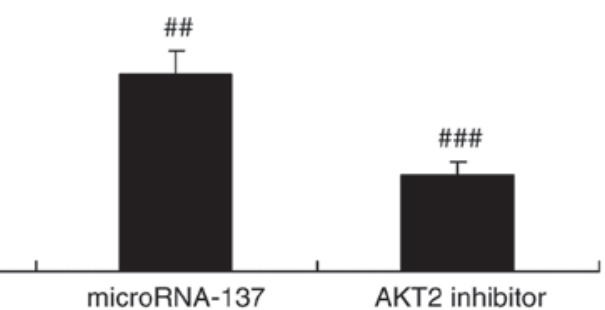

(
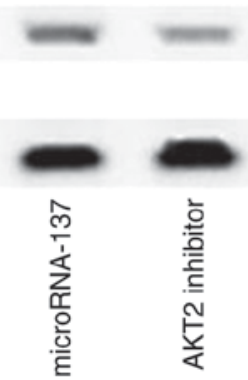

Cyclin DI

GAPDH

Figure 9. Effect of an AKT2 inhibitor on Cyclin D1 protein expression in A549 and H520 cells treated with cisplatin following downregulation of microRNA-137. (A) Densitometric and (B) western blot analysis of the effect of an AKT2 inhibitor on Cyclin D1 protein expression in A549 cells treated with cisplatin following downregulation of microRNA-137. (C) Densitometric and (D) western blot analysis of the effect of an AKT2 inhibitor on Cyclin D1 protein expression in H520 cells treated with cisplatin following downregulation of microRNA-137. ${ }^{\#} \mathrm{P}<0.01$ compared with microRNA-Negative group; \#\# $\mathrm{P}<0.01$ compared with microRNA-137 group. Negative, negative control group; microRNA-137, microRNA-137 group; AKT2 inhibitor, microRNA-137+MK2206 group.

numerous steps and the participation of various genes (23). The regulation of cell proliferation and apoptosis serves an important role in the progression of numerous stages in the development of NSCLC (24). Apoptosis is also known as programmed cell death and is crucial in the development and stabilization of multi-cellular organisms (25). In particular, it 
serves an important role in embryonic development modeling, the regulation of cells and the elimination of cells with potential risks. Programmed cell death primarily includes two pathways, namely the death receptor pathway (exogenous pathway) and the mitochondrial pathway (endogenous pathway) (24). By virtue of a series of molecules and biochemical pathways, the common 'central processing unit molecule', namely excitation of Caspases belonging to two pathways, induces degradation of associated substrates in the nucleus and cytoplasm. Bax is an important regulatory factor of apoptosis. The Bax gene belongs to the $\mathrm{B}$ cell lymphoma-2 family and serves a role in the promotion of apoptosis (26). The results of the present study demonstrated that the overexpression of microRNA-137 significantly induced the expression of Bax protein in A549 and H520 cells treated with cisplatin.

Research on cell strains demonstrated that p-AKT is able to upregulate Cyclin D1 expression through multiple pathways and that it promotes cell proliferation-it phosphorylates downstream mTOR, which phosphorylates $\mathrm{P} 70^{\mathrm{s} 6 \mathrm{k}}(27)$. The activated $\mathrm{P} 70^{\mathrm{s} 6 \mathrm{k}}$ mediates ribosomal protein. The cell cycle G1-S phases transform the crucial translation of miRNAs (28). By phosphorylating GSK3 and resisting the degradation of $\beta$-Catenin, $\beta$-Catenin accumulation in the cytoplasm enters into the nucleus and lymphocyte enhancer to launch the transcription of cytokines (LEF/TCF), as well as to upregulate Cyclin D1 expression (29). In conclusion, the results of the present study revealed that overexpression of microRNA-137 significantly suppressed p-AKT2 protein expression and inhibited Cyclin D1 protein expression in A549 and H520 cells treated with cisplatin.

A previous study on AKT revealed that AKT may promote the proliferation of tumor cells by phosphorylating multiple downstream substrates, restraining apoptosis, improving the anoxia tolerance of cells, promoting angiogenesis, promoting the invasion and metastasis of tumor cells, and promoting resistance to chemotherapy and radiotherapy (17). In numerous tumor tissues, AKT is overexpressed and exhibits increased activity (expressed as phosphorylation). AKT2 is an important subtype of the AKT gene and has already been confirmed to be an oncogene (30). In multiple types of tumor tissues there is an overexpression or increased activity of the AKT2 protein. It has been proven that the PI3K/AKT pathway participates in the generation and development of multiple types of tumors (30). The results of the present study revealed that the inhibition of AKT2 also increased caspase-3 activity and Bax protein expression, and suppressed Cyclin D1 protein expression in A549 and H520 cells treated with cisplatin following overexpression of microRNA-137.

In conclusion, the results of the present study revealed that the overexpression of microRNA-137 inhibited cell proliferation and increased the caspase-3 activity of A549 and H520 cells treated with cisplatin through suppression of Cyclin D1 and activation of the Bax pathway by targeting AKT2. Therefore, microRNA-137-regulated AKT2 may be a potential therapeutic strategy for the treatment of patients with NSCLC using cisplatin.

\section{Acknowledgements}

Not applicable.

\section{Funding}

No funding was received.

\section{Availability of data and materials}

The analyzed data sets generated during the study are available from the corresponding author on reasonable request.

\section{Authors' contributions}

ZL designed the experiment. MW, SW, MY, ZL, TS and CD performed the experiment. ZL and MW analyzed the data. ZL wrote the manuscript.

\section{Ethics approval and consent to participate}

The experimental protocols were approved by the Ethics Committees of Jiangmen Central Hospital and written informed consent was obtained from all participants.

\section{Consent for publication}

All patients provided consent for publication.

\section{Competing interests}

The authors declare that they have no competing interests.

\section{References}

1. Zhao Z, Liao H and Ju Y: Effect of compound Kushen injection on T-cell subgroups and natural killer cells in patients with locally advanced non-small-cell lung cancer treated with concomitant radiochemotherapy. J Tradit Chin Med 36: 14-18, 2016.

2. Videtic GM, Hu C, Singh AK, Chang JY, Parker W, Olivier KR, Schild SE, Komaki R, Urbanic JJ and Choy H: A randomized phase 2 study comparing 2 stereotactic body radiation therapy schedules for medically inoperable patients with stage I peripheral non-small cell lung cancer: NRG oncology RTOG 0915 (NCCTG N0927). Int J Radiat Oncol Biol Phys 93: 757-764, 2015.

3. Zinner RG, Obasaju CK, Spigel DR, Weaver RW, Beck JT, Waterhouse DM, Modiano MR, Hrinczenko B, Nikolinakos PG, Liu J, et al: PRONOUNCE: Randomized, open-label, phase III study of first-line pemetrexed + carboplatin followed by maintenance pemetrexed versus paclitaxel + carboplatin + bevacizumab followed by maintenance bevacizumab in patients ith advanced nonsquamous non-small-cell lung cancer. J Thorac Oncol 10: 134-142, 2015.

4. Semrau S, Zettl H, Hildebrandt G, Klautke G and Fietkau R: Older patients with inoperable non-small cell lung cancer: Long-term survival after concurrent chemoradiotherapy. Strahlenther Onkol 190: 1125-1132, 2014.

5. Hu W and Zhang Z: A phase II clinical study of using nab-paclitaxel as second-line chemotherapy for Chinese patients with advanced non-small cell lung cancer. Med Oncol 32: 498, 2015.

6. McKenna E, Traganos F, Zhao H and Darzynkiewicz Z: Persistent DNA damage caused by low levels of mitomycin $\mathrm{C}$ induces irreversible cell senescence. Cell Cycle 11: 3132-3140, 2012.

7. Li Y, Qi K, Zu L, Wang M, Wang Y and Zhou Q: Anti-apoptotic brain and reproductive organ-expressed proteins enhance cisplatin resistance in lung cancer cells via the protein kinase B signaling pathway. Thorac Cancer 7: 190-198, 2016.

8. Reck M, Paz-Ares LG, de Marinis F, Molinier O, Sahoo TP, Laack E, John W, Zimmermann AH, Visseren-Grul C and Gridelli C: PARAMOUNT: Descriptive subgroup analyses of final overall survival for the phase III study of maintenance pemetrexed versus placebo following induction treatment with pemetrexed plus cisplatin for advanced nonsquamous non-small-cell lung cancer. J Thorac Oncol 9: 205-213, 2014. 
9. Yang L, Yuan Y, Fu C, Xu X, Zhou J, Wang S, Kong L, Li Z, Guo Q and Wei L: LZ-106, a novel analog of enoxacin, inducing apoptosis via activation of ROS-dependent DNA damage response in NSCLCs. Free Radic Biol Med 95: 155-168, 2016.

10. Petrelli F and Barni S: Non-cancer-related mortality after cisplatin-based adjuvant chemotherapy for non-small cell lung cancer: A study-level meta-analysis of 16 randomized trials. Med Oncol 30: 641, 2013

11. Niho S, Kenmotsu H, Sekine I, Ishii G, Ishikawa Y, Noguchi M, Oshita F, Watanabe S, Nakajima R, Tada H and Nagai $K$ : Combination chemotherapy with irinotecan and cisplatin for large-cell neuroendocrine carcinoma of the lung: A multicenter phase II study. J Thorac Oncol 8: 980-984, 2013.

12. Li Q, Yang Z, Chen M and Liu Y: Downregulation of microRNA-196a enhances the sensitivity of non-small cell lung cancer cells to cisplatin treatment. Int J Mol Med 37: 1067-1074, 2016.

13. Wu L, Pu X, Wang Q, Cao J, Xu F, Xu LI and Li K: miR-96 induces cisplatin chemoresistance in non-small cell lung cancer cells by downregulating SAMD9. Oncol Lett 11: 945-952, 2016.

14. Zhang Z, Zhang L, Yin ZY, Fan XL, Hu B, Wang LQ and Zhang D: miR-107 regulates cisplatin chemosensitivity of A549 non small cell lung cancer cell line by targeting cyclin dependent kinase 8. Int J Clin Exp Pathol 7: 7236-7241, 2014.

15. Berghmans T, Ameye L, Lafitte JJ, Colinet B, Cortot A, CsToth I, Holbrechts S, Lecomte J, Mascaux C, Meert AP, et al: Prospective validation obtained in a similar group of patients and with similar high throughput biological tests failed to confirm signatures for prediction of response to chemotherapy and survival in advanced NSCLC: A prospective study from the european lung cancer working party. Front Oncol 4: 386, 2015.

16. Hu W, Jin P and Liu W: Periostin contributes to cisplatin resistance in human non-small cell lung cancer A549 cells via activation of Stat 3 and Akt and upregulation of survivin. Cell Physiol Biochem 38: 1199-1208, 2016.

17. Wu HH, Wu JY, Cheng YW, Chen CY, Lee MC, Goan YG and Lee H: cIAP2 upregulated by E6 oncoprotein via epidermal growth factor receptor/phosphatidylinositol 3-kinase/AKT pathway confers resistance to cisplatin in human papillomavirus 16/18-infected lung cancer. Clin Cancer Res 16: 5200-5210, 2010.

18. Hu B, Zhang Y, Jia L, Wu H, Fan C, Sun Y, Ye C, Liao M and Zhou J: Binding of the pathogen receptor HSP90AA1 to avibirnavirus VP2 induces autophagy by inactivating the AKT-MTOR pathway. Autophagy 11: 503-515, 2015.

19. Livak KJ and Schmittgen TD: Analysis of relative gene expression data using real-time quantitative PCR and the 2(-Delta Delta C(T)) method. Methods 25: 402-408, 2001.
20. Sebastian M, Papachristofilou A, Weiss C, Früh M, Cathomas R, Hilbe W, Wehler T, Rippin G, Koch SD, Scheel B, et al: Phase Ib study evaluating a self-adjuvanted mRNA cancer vaccine $\left(\right.$ RNActive $^{\circledR}$ ) combined with local radiation as consolidation and maintenance treatment for patients with stage IV non-small cell lung cancer. BMC Cancer 14: 748, 2014.

21. Neault M, Mallette FA and Richard S: miR-137 modulates a tumor suppressor Network-inducing senescence in pancreatic cancer cells. Cell Rep 14: 1966-1978, 2016.

22. Shin KK, Kim YS, Kim JY, Bae YC and Jung JS: miR-137 controls proliferation and differentiation of human adipose tissue stromal cells. Cell Physiol Biochem 33: 758-768, 2014.

23. Chen B, Wang X, Zhao W and Wu J: Klotho inhibits growth and promotes apoptosis in human lung cancer cell line A549. J Exp Clin Cancer Res 29: 99, 2010.

24. Fang C, Zhang J, Qi D, Fan X, Luo J, Liu L and Tan Q Evodiamine induces $\mathrm{G} 2 / \mathrm{M}$ arrest and apoptosis via mitochondrial and endoplasmic reticulum pathways in $\mathrm{H} 446$ and $\mathrm{H} 1688$ human small-cell lung cancer cells. PLoS One 9: e115204, 2014.

25. Chougule M, Patel AR, Sachdeva P, Jackson T and Singh M: Anticancer activity of Noscapine, an opioid alkaloid in combination with Cisplatin in human non-small cell lung cancer. Lung Cancer 71: 271-282, 2011

26. Peng Y, Wang L, Qing Y, Li C, Ren T, Li Q, Li M, Zhang S, Shan J, Wang G, et al: Polymorphisms of BCL2 and BAX Genes associate with outcomes in advanced Non-small cell lung cancer patients treated with platinum-based Chemotherapy. Sci Rep 5: 17766, 2015.

27. Żuryń A, Litwiniec A, Safiejko-Mroczka B, Klimaszewska-Wiśniewska A, Gagat M, Krajewski A, Gackowska L and Grzanka D: The effect of sulforaphane on the cell cycle, apoptosis and expression of cyclin D1 and p21 in the A549 non-small cell lung cancer cell line. Int J Oncol 48: 2521-2533, 2016

28. Chen Y, Cao Y, Yang D, Li K, Wang Z, Zhu J, Bunjhoo H, Xiong $\mathrm{S}, \mathrm{Xu} \mathrm{Y}$ and Xiong $\mathrm{W}$ : Increase of the therapeutic effect on non-small-cell lung cancer cells with combination treatment of shRNA against Cyclin D1 and Bcl-xL in vitro. Exp Ther Med 3: 255-260, 2012

29. Singh T, Prasad R and Katiyar SK: Inhibition of class I histone deacetylases in non-small cell lung cancer by honokiol leads to suppression of cancer cell growth and induction of cell death in vitro and in vivo. Epigenetics 8: 54-65, 2013

30. Fu QF, Liu Y, Fan Y, Hua SN, Qu HY, Dong SW, Li RL, Zhao MY, Zhen Y, Yu XL, et al: Alpha-enolase promotes cell glycolysis, growth, migration, and invasion in non-small cell lung cancer through FAK-mediated PI3K/AKT pathway. J Hematol Oncol 8: 22, 2015. 